\title{
The lowest vibration spectra of multi-component structures with contrast material properties
}

\author{
J. Kaplunov*, D.A. Prikazchikov, L.A. Prikazchikova, O. Sergushova \\ School of Computing and Mathematics, Keele University, \\ Keele, Staffordshire, ST5 5BG, UK
}

\begin{abstract}
The paper is concerned with the lowest vibration modes of multi-component rods and cylinders with alternating high contrast material properties of the components. It is demonstrated that these modes correspond to almost rigid body motions of the "stiffer" components. A general perturbation scheme is developed. At leading order polynomial frequency equations are derived, along with linear algebraic equations for the associated eigenforms. In addition, for multi-component rods, higher order corrections are also obtained. Examples of three- and five-component structures are presented, illustrating the efficiency of the proposed approach.

Keywords: Multi-component, contrast, lowest modes, perturbation, rod, cylinder.
\end{abstract}

\section{Introduction}

Composite materials with high-contrast properties find numerous applications in modern engineering, including in particular sandwich structures as photovoltaic panels [1] and laminated glass [2], energy scavenging devices [3], smart 5 periodic structures [4, and acoustic metamaterial components [5]. In addition, we mention [6], reporting on the recent state of art within the area of laminated plates and sandwich structures.

*Corresponding author
Email address: j.kaplunov@keele.ac.uk (J. Kaplunov)

Preprint submitted to Journal of Sound and Vibration

January 6, 2019 
There are two main methodologies for theoretical analysis of high-contrast composite materials. The first one deals with asymptotic homogenisation of high-contrast periodic media at various level of mathematical rigour, see e.g. [7, 8], 9], and [10. Another research strand is oriented mainly towards twoor three-component structures. For example, in [11], the low-frequency vibrations of a three-component elastic rod are studied, revealing the conditions on material parameters resulting in the so-called "global" low-frequency regime, associated with polynomial eigenforms, along with the "local" low-frequency regime, for which the displacements of certain components demonstrate oscillatory behaviour. Vibrations of a two-component string on an elastic foundation over the near-cut-off frequency range are analysed in [12. The current contribution aims at composite structures involving arbitrary number of components with high contrast properties, in a sense bridging the gap between the aforementioned setups. We also mention here the analogy between the asymptotic procedures for periodic media and layered thin structures, addressed in [13].

The proposed approach relies on the concept of "almost rigid body motion", see e.g. [11. In this case the stiffer components, subject to free ends boundary conditions, perform rigid body motion at leading order. At the same time the softer parts, subject to fixed ends boundary conditions, undergo quasi-static deformation, which is almost homogeneous for a rod. The observations in [11] are also relevant for imposing conditions on material parameters of a threelayered strongly inhomogeneous plate, leading to the first shear cut-off tending 30 to zero and thus justifying two-mode low-frequency approximations, see [14, and [15.

In the present paper we study multi-component structures with arbitrary number of components. More specifically, we focus on longitudinal deformations of rods and axisymmetric antiplane motions of cylinders. The related 35 exact solutions are rather involved, especially for multi-layered cylinders, with a lengthy transcendental frequency equation containing Bessel functions, hence, motivating an asymptotic consideration.

A perturbation procedure is established. At leading order, we observe rigid 
body motion of the stiffer components along with static deformation of softer parts. It is remarkable that for a rod softer parts undergo almost homogeneous deformation, whereas for a cylinder a logarithmic behaviour for displacement is observed due to the effect of curvature. At next order, we establish a polynomial frequency equation, with the polynomial order being equal to the number of stiffer components. Simultaneously, we derive linear algebraic equations for

45 the sought for eigenforms. In addition, for rods, proceeding to higher orders, we derive refined two-term expansions for the eigenfrequencies and eigenforms. Illustrative examples for three- and five- component rods and cylinders are presented.

\section{Perturbation scheme for a multi-component rod}

Consider an $n$-component rod composed of alternating stiff and soft parts of arbitrary lengths $l_{i}, i=1, \ldots n$, see Figure 1. Assume that the Young's modulus of the stiffer parts, $E_{1}$, is much greater than that of the softer components, $E_{2}$, i.e.

$$
\varepsilon=\frac{E_{2}}{E_{1}} \ll 1
$$

${ }_{50}$ is a small parameter. For the sake of simplicity, we also assume that the densities of stiffer and softer components, $\rho_{1}$ and $\rho_{2}$ are related as $\rho_{2} / \rho_{1} \sim \varepsilon$.

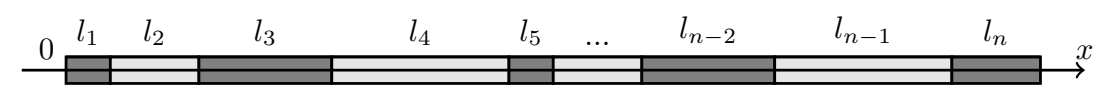

Figure 1: A multi-component piecewise-homogeneous rod.

The equations of motion of the components are given by

$$
\frac{\mathrm{d}^{2} u_{i}}{\mathrm{~d} x^{2}}+\frac{\omega^{2}}{c_{m}^{2}} u_{i}=0, \quad i=1,2, \ldots, n ; \quad m=1,2,
$$

where $u_{i}$ are displacements, $x$ is longitudinal coordinate, $\omega$ is vibration frequency, $c_{m}=c_{1}$ or $c_{m}=c_{2}$ for the stiff and soft components, respectively, with $c_{m}=\sqrt{E_{m} / \rho_{m}}$, denoting longitudinal wave speeds. 
Let us define local dimensionless coordinates and frequencies by

$$
X_{i}=\frac{x}{l_{i}}, \quad \text { and } \quad \Omega_{i}=\frac{\omega l_{i}}{c_{m}} .
$$

In addition, the relation

$$
\Omega_{i}=L_{j}^{i} \Omega_{j},
$$

is used for the stiff components, where $L_{j}^{i}=l_{i} / l_{j}$. Also, we introduce dimensionless quantities

$$
b_{i}=\frac{1}{l_{i}} \sum_{n=0}^{i-1} l_{n}, \quad i=1,2, \ldots, n,
$$

such that

$$
b_{i} \leq X_{i} \leq b_{i}+1 .
$$

In this section we set $l_{0}=0$, while in Section 4 below we will have $l_{0}>0$. The equations of motion (1) rewritten in dimensionless form become

$$
\frac{\mathrm{d}^{2} u_{i}}{\mathrm{~d} X_{i}^{2}}+\Omega_{i}^{2} u_{i}=0 .
$$

The continuity conditions at the interfaces are given by

$$
\left.u_{i}\right|_{X_{i}=b_{i}+1}=\left.u_{i+1}\right|_{X_{i+1}=b_{i+1}}
$$

and

$$
\left.\frac{\mathrm{d} u_{i}}{\mathrm{~d} X_{i}}\right|_{X_{i}=b_{i}+1}=\left.\varepsilon^{j} L_{i+1}^{i} \frac{\mathrm{d} u_{i+1}}{\mathrm{~d} X_{i+1}}\right|_{X_{i+1}=b_{i+1}},
$$

where $j=1$ if the $i$-th component is stiff, and $j=-1$ if it is soft.

For the sake of definiteness, in this section we restrict ourselves to the case in which the ends of the outer stiffer components $(i=1$ and $i=n)$ are tractionfree, i.e.

$$
\left.\frac{\mathrm{d} u_{1}}{\mathrm{~d} X_{1}}\right|_{X_{1}=0}=\left.\frac{\mathrm{d} u_{n}}{\mathrm{~d} X_{n}}\right|_{X_{n}=b_{n}+1}=0 .
$$

Thus, the overall number of components $n$ is an odd number, and $n \geq 3$.

The objective of the paper is to tackle the frequency range $\Omega_{1}^{2} \sim \Omega_{2}^{2} \sim \ldots \sim$ $\Omega_{n}^{2} \sim \varepsilon$, which has been referred to in [11] as the global low-frequency one. Let us expand frequency parameters $\Omega_{i}$ and displacements $u_{i}$ in the asymptotic series

$$
\Omega_{i}^{2}=\varepsilon\left(\Omega_{i 0}^{2}+\varepsilon \Omega_{i 1}^{2}+\ldots\right)
$$


and

$$
u_{i}=u_{i 0}+\varepsilon u_{i 1}+\ldots
$$

First, we deduce the leading order displacements for all of the $n_{1}=\frac{n+1}{2}$ stiff inner components by substituting $(10)$ and $(11)$ into $(6)$ and $(8)$. Then, we obtain boundary value problems for the equations

$$
\frac{\mathrm{d}^{2} u_{i 0}}{\mathrm{~d} X_{i}^{2}}=0
$$

with $i=2 p-1, p=1,2, \ldots, n_{1}$, subject to the homogeneous Neumann boundary conditions

$$
\left.\frac{\mathrm{d} u_{i 0}}{\mathrm{~d} X_{i}}\right|_{X_{i}=b_{i}}=\left.\frac{\mathrm{d} u_{i 0}}{\mathrm{~d} X_{i}}\right|_{X_{i}=b_{i}+1}=0 .
$$

Therefore, for the displacements of the stiff components we have uniform static variations, corresponding to the rigid body motions, i.e.

$$
u_{i 0}=C_{i, 0}=\text { const }
$$

The leading order boundary value problems for the $n_{2}=\frac{n-1}{2}$ soft components follow from the substitution of $(10)$ and $(11)$ into $(6)$ and $(7)$. Then, taking into account the solution (14), we obtain the inhomogeneous Dirichlet boundary conditions

$$
\left.u_{i 0}\right|_{X_{i}=b_{i}}=C_{i-1,0},\left.\quad u_{i 0}\right|_{X_{i}=b_{i}+1}=C_{i+1,0}
$$

for the same equation (12), with $i=2 p, p=1,2, \ldots, n_{2}$, governing now the equilibrium of the soft components. As a result, we arrive at the following linear displacement variations of the soft components

$$
u_{i 0}=C_{i-1,0}+\left(C_{i+1,0}-C_{i-1,0}\right)\left(X_{i}-b_{i}\right)
$$

corresponding to their homogeneous deformations.

Let us now proceed to the next order problems for the stiff components, having the equations

$$
\frac{\mathrm{d}^{2} u_{i 1}}{\mathrm{~d} X_{i}^{2}}+\Omega_{i 0}^{2} u_{i 0}=0, \quad i=2 p-1, p=1,2, \ldots, n_{1},
$$


with the Neumann boundary conditions for the inner parts given by

$$
\begin{aligned}
& \left.\frac{\mathrm{d} u_{i 1}}{\mathrm{~d} X_{i}}\right|_{X_{i}=b_{i}}=L_{i-1}^{i}\left(C_{i, 0}-C_{i-2,0}\right), \\
& \left.\frac{\mathrm{d} u_{i 1}}{\mathrm{~d} X_{i}}\right|_{X_{i}=b_{i}+1}=L_{i+1}^{i}\left(C_{i+2,0}-C_{i, 0}\right) .
\end{aligned}
$$

For the outer parts boundary conditions are written as

$$
\left.\frac{\mathrm{d} u_{11}}{\mathrm{~d} X_{1}}\right|_{X_{1}=0}=0,\left.\quad \frac{\mathrm{d} u_{11}}{\mathrm{~d} X_{1}}\right|_{X_{1}=1}=L_{2}^{1}\left(C_{3,0}-C_{1,0}\right),
$$

and

$$
\left.\frac{\mathrm{d} u_{n 1}}{\mathrm{~d} X_{n}}\right|_{X_{n}=b_{n}}=L_{n-1}^{n}\left(C_{n, 0}-C_{n-2,0}\right),\left.\quad \frac{\mathrm{d} u_{n 1}}{\mathrm{~d} X_{n}}\right|_{X_{n}=b_{n}+1}=0 .
$$

We integrate equations (17) over $X_{i}\left(b_{i} \leq X_{i} \leq b_{i}+1\right)$, taking into account (14), resulting in

$$
\left.\frac{\mathrm{d} u_{i 1}}{\mathrm{~d} X_{i}}\right|_{b_{i}} ^{b_{i}+1}=-\Omega_{i 0}^{2} C_{i, 0} .
$$

Next, combining the formulae (18)-(21), we obtain for each of the stiffer components

$$
\begin{aligned}
& C_{1,0} \Omega_{10}^{2}=L_{2}^{1}\left(C_{1,0}-C_{3,0}\right) \\
& \quad \vdots \\
& C_{i, 0} \Omega_{i 0}^{2}=\left(L_{i-1}^{i}+L_{i+1}^{i}\right) C_{i, 0}-L_{i-1}^{i} C_{i-2,0}-L_{i+1}^{i} C_{i+2,0}, \\
& \quad \vdots \\
& C_{n, 0} \Omega_{n 0}^{2}=L_{n-1}^{n}\left(C_{n, 0}-C_{n-2,0}\right)
\end{aligned}
$$

where $i=2 p-1, p=1,2, \ldots, n_{1}$, and the frequency parameters are related to each other by (3). The derived relations result in the frequency equation in the form of a polynomial of order $n_{1}$ in one of the frequency parameters above, e.g. $\Omega_{10}^{2}$, see also examples below. Note that in the special case $C_{i, 0}=0$ the system (22) degenerates, see the example in subsection 3.2.

The eigenforms for the stiff components then come from linear equations (22), namely

$$
u_{i 1}=A_{i, 1} X_{i}^{2}+B_{i, 1} X_{i}+C_{i, 1}, \quad i=2 p-1, p=1,2, \ldots, n_{1},
$$


where the constants are defined as

$$
A_{i, 1}=-\frac{1}{2} \Omega_{i 0}^{2} u_{i 0},
$$

and

$$
\begin{aligned}
B_{1,1} & =0 \\
\quad & \\
B_{j, 1} & =L_{j+1}^{j}\left(C_{j+2,0}-C_{j, 0}\right)+\Omega_{j 0}^{2} u_{j 0}\left(b_{j}+1\right), \\
\quad & \\
B_{n, 1} & =\Omega_{n 0}^{2} u_{n 0}\left(b_{n}+1\right),
\end{aligned}
$$

$j=2 p-1, p=2, \ldots, n_{1}-1$. Unknown constants $C_{i, 1}$ in 23 are to be found from the next order problem.

Consider the Dirichlet boundary value problem for the soft components of the rod

$$
\frac{\mathrm{d}^{2} u_{i 1}}{\mathrm{~d} X_{i}^{2}}+\Omega_{i 0}^{2} u_{i 0}=0, \quad i=2 p, p=1,2, \ldots, n_{2},
$$

subject to the boundary conditions

$$
\begin{aligned}
& \left.u_{i 1}\right|_{X_{i}=b_{i}}=A_{i-1,1}\left(b_{i-1}+1\right)^{2}+B_{i-1,1}\left(b_{i-1}+1\right)+C_{i-1,1}, \\
& \left.u_{i 1}\right|_{X_{i}=b_{i}+1}=A_{i+1,1} b_{i+1}^{2}+B_{i+1,1} b_{i+1}+C_{i+1,1} .
\end{aligned}
$$

Solving this problem we obtain for the soft components

$$
u_{i 1}=N_{i, 1} X_{i}^{3}+F_{i, 1} X_{i}^{2}+\left(G_{i, 1}+C_{i+1,1}-C_{i-1,1}\right) X_{i}+H_{i, 1},
$$

where

$$
\begin{aligned}
N_{i, 1} & =-\frac{1}{6} \Omega_{i 0}^{2}\left(C_{i+1,0}-C_{i-1,0}\right) \\
F_{i, 1} & =-\frac{1}{2} \Omega_{i 0}^{2}\left(C_{i-1,0}-\left(C_{i+1,0}-C_{i-1,0}\right) b_{i}\right), \\
G_{i, 1} & =A_{i+1,1} b_{i+1}^{2}-A_{i-1,1}\left(b_{i-1}+1\right)^{2}+B_{i+1,1} b_{i+1}-B_{i-1,1}\left(b_{i-1}+1\right) \\
& -N_{i, 1}\left(3 b_{i}^{2}+3 b_{i}+1\right)-F_{i, 1}\left(2 b_{i}+1\right), \\
H_{i, 1} & =A_{i+1,1} b_{i+1}^{2}+B_{i+1,1} b_{i+1}+C_{i+1,1}-N_{i, 1}\left(b_{i}+1\right)^{3}-F_{i, 1}\left(b_{i}+1\right)^{2} \\
& -\left(G_{i, 1}+C_{i+1,1}-C_{i-1,1}\right)\left(b_{i}+1\right),
\end{aligned}
$$


In order to determine unknown constants $C_{i, 1}$, we have to proceed to the next order problem for the stiff components, namely

$$
\frac{\mathrm{d}^{2} u_{i 2}}{\mathrm{~d} X_{i}^{2}}+\Omega_{i 0}^{2} u_{i 1}+\Omega_{i 1}^{2} u_{i 0}=0, \quad i=2 p-1, p=1,2, \ldots, n_{1}
$$

subject to the Neumann boundary conditions for the inner components

$$
\begin{aligned}
& \left.\frac{\mathrm{d} u_{i 2}}{\mathrm{~d} X_{i}}\right|_{X_{i}=b_{i}}=L_{i-1}^{i}\left(3 N_{i-1,1}\left(b_{i-1}+1\right)^{2}+2 F_{i-1,1}\left(b_{i-1}+1\right)+G_{i-1,1}\right), \\
& \left.\frac{\mathrm{d} u_{i 2}}{\mathrm{~d} X_{i}}\right|_{X_{i}=b_{i}+1}=L_{i+1}^{i}\left(3 N_{i+1,1} b_{i+1}^{2}+2 F_{i+1,1} b_{i+1}+G_{i+1,1}\right),
\end{aligned}
$$

and for the outer components

$$
\begin{aligned}
& \left.\frac{\mathrm{d} u_{12}}{\mathrm{~d} X_{1}}\right|_{X_{1}=0}=0, \\
& \left.\frac{\mathrm{d} u_{12}}{\mathrm{~d} X_{1}}\right|_{X_{1}=1}=L_{2}^{1}\left(3 N_{2,1} b_{2}^{2}+2 F_{2,1} b_{2}+G_{2,1}\right),
\end{aligned}
$$

and

$$
\begin{aligned}
& \left.\frac{\mathrm{d} u_{n 2}}{\mathrm{~d} X_{n}}\right|_{X_{n}=b_{n}}=L_{n-1}^{n}\left(3 N_{n-1,1}\left(b_{n-1}+1\right)^{2}+2 F_{n-1,1}\left(b_{n-1}+1\right)+G_{n-1,1}+C_{n, 1}-C_{n-2,1}\right), \\
& \left.\frac{\mathrm{d} u_{n 2}}{\mathrm{~d} X_{n}}\right|_{X_{n}=b_{n}+1}=0 .
\end{aligned}
$$


Integrating (30), we arrive at simultaneous equations similar to 22 . They are

$$
\begin{aligned}
C_{1,0} \Omega_{11}^{2}= & -L_{2}^{1}\left(3 N_{2,1} b_{2}^{2}+2 F_{2,1} b_{2}+G_{2,1}+C_{3,1}\right)-\frac{1}{3} A_{1,1} \Omega_{10}^{2} \\
\vdots & \\
C_{i, 0} \Omega_{i 1}^{2}= & -L_{i+1}^{i}\left\{\left(A_{i+2,1} b_{i+2}^{2}-A_{i, 1}\left(b_{i}+1\right)^{2}+B_{i+2,1} b_{i+2}-B_{i, 1}\left(b_{i}+1\right)\right.\right. \\
& \left.-N_{i+1,1}\left(3 b_{i+1}+1\right)-F_{i+1,1}+C_{i+2,1}-C_{i, 1}\right\} \\
& +L_{i-1}^{i}\left\{A_{i, 1} b_{i}^{2}-A_{i-2,1}\left(b_{i-2}+1\right)^{2}+B_{i, 1} b_{i}-B_{i-2,1}\left(b_{i-2}+1\right)\right. \\
& \left.+N_{i-1,1}\left(3 b_{i-1}+2\right)+F_{i-1,1}+C_{i, 1}-C_{i-2,1}\right\} \\
& -\Omega_{i 0}^{2}\left(\frac{1}{3} A_{i, 1}\left(3 b_{i}^{2}+3 b_{i}+1\right)+\frac{1}{2} B_{i, 1}\left(2 b_{i}+1\right)+C_{i, 1}\right) \\
\vdots \quad & \\
C_{n, 0} \Omega_{n 1}^{2}= & L_{n-1}^{n}\left\{3 N_{n-1,1}\left(b_{n-1}+1\right)^{2}+2 F_{n-1,1}\left(b_{n-1}+1\right)\right. \\
& \left.+G_{n-1,1}+C_{n, 1}-C_{n-2,1}\right\} \\
& -\Omega_{n 0}^{2}\left(\frac{1}{3} A_{n, 1}\left(3 b_{n}^{2}+3 b_{n}+1\right)+\frac{1}{2} B_{n, 1}\left(2 b_{n}+1\right)+C_{n, 1}\right)
\end{aligned}
$$

65 through (3).

Setting, for example, $C_{1,1}=0$ we can find the rest of the constants $C_{i, 1}$ from (31) together with the correction to the frequency parameter, say, expressed in terms of $\Omega_{11}^{2}$.

\section{Three- and five-component rods}

\subsection{Three-component rod}

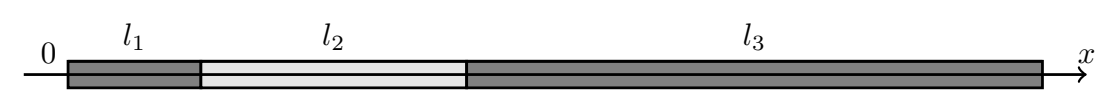

Figure 2: A three-component piecewise-homogeneous rod.

As an example, consider first a three-component rod with two stiff and one soft components $\left(n_{1}=2, n_{2}=1\right)$, shown in Figure 2. In this case we have from 
(22) and also (3) for the stiff parts

$$
\begin{aligned}
& C_{1,0} \Omega_{10}^{2}=L_{2}^{1}\left(C_{1,0}-C_{3,0}\right), \\
& C_{3,0} \Omega_{30}^{2}=L_{2}^{3}\left(C_{3,0}-C_{1,0}\right),
\end{aligned}
$$

together with

$$
\Omega_{10}=L_{3}^{1} \Omega_{30} .
$$

These lead to a bi-quadratic frequency equation given by

$$
\Omega_{10}^{2}\left(\Omega_{10}^{2}-L_{2}^{1}\left(1+L_{3}^{1}\right)\right)=0 .
$$

Its solution can be written as

$$
\Omega_{10}^{2}=k L_{2}^{1},
$$

where $k=0$ or $k=1+L_{3}^{1}$. The last result coincides with that in [1]. We also have the relation

$$
C_{3,0}=(1-k) C_{1,0} .
$$

Using (14) and (16) with 36 we can determine the displacement profiles in terms of one constant only, e.g. $C_{1,0}$. Then, the eigenforms become

$$
\begin{aligned}
& u_{10}=C_{1,0}, \\
& u_{20}=C_{1,0}\left(1-k\left(X_{2}-b_{2}\right)\right), \\
& u_{30}=C_{1,0}(1-k) .
\end{aligned}
$$

Clearly, the last expressions are exact at $k=0$ and correspond to rigid body motion with zero frequency. Proceeding with the next order approximation at $k=1+L_{3}^{1}$, see [31), we arrive at a correction to the sought for eigenfrequencies given by

$$
\begin{aligned}
\Omega_{11}^{2}=\frac{L_{2}^{1}}{6}\left\{k^{2} L_{2}^{1}+3 k L_{2}^{1}\left(-1+b_{3}\left(b_{3}+2\right)(k-1)\right.\right. & \left.\left(L_{1}^{3}\right)^{2}\right) \\
& \left.+c^{2} L_{1}^{2} k(k-3)-6 C_{3,1}\right\},
\end{aligned}
$$


where $c=c_{1} / c_{2}$ and the related eigenforms are expressed through a single constant as

$$
\begin{aligned}
& u_{11}=A_{1,1} X_{1}^{2}, \\
& u_{21}=N_{2,1} X_{2}^{3}+F_{2,1} X_{2}^{2}+\left(G_{2,1}+C_{3,1}\right) X_{2}+H_{2,1}, \\
& u_{31}=A_{3,1} X_{3}^{2}+B_{3,1} X_{3}+C_{3,1},
\end{aligned}
$$

where

$$
\begin{aligned}
& A_{1,1}=-\frac{1}{2} \Omega_{10}^{2} C_{1,0}, \\
& A_{3,1}=-\frac{1}{2}(1-k) \Omega_{30}^{2} C_{1,0}, \\
& B_{3,1}=(1-k)\left(b_{3}+1\right) \Omega_{30}^{2} C_{1,0}, \\
& N_{2,1}=\frac{1}{6} k \Omega_{20}^{2} C_{1,0}, \\
& F_{2,1}=-\frac{1}{2}\left(1+k b_{2}\right) \Omega_{20}^{2} C_{1,0}, \\
& G_{2,1}=A_{3,1} b_{3}^{2}-A_{1,1}\left(b_{1}+1\right)^{2}+B_{3,1} b_{3}-N_{2,1}\left(3 b_{2}^{2}+3 b_{2}+1\right)-F_{2,1}\left(2 b_{2}+1\right), \\
& H_{2,1}=A_{3,1} b_{3}^{2}+B_{3,1} b_{3}-C_{3,1} b_{2}-N_{2,1}\left(b_{2}+1\right)^{3}-F_{2,1}\left(b_{2}+1\right)^{2}-G_{2,1}\left(b_{2}+1\right),
\end{aligned}
$$

and $C_{3,1}$ is given in Appendix A.

For a regular three-component $\operatorname{rod} l_{i}=l, i=1,2,3$, we have the two-term expansions $(k=2)$

$$
\Omega_{1}^{2}=2 \varepsilon-\frac{\varepsilon^{2}}{3}\left(c^{2}+4\right)+\ldots
$$

with

$$
\begin{aligned}
& u_{1}=1-\varepsilon X_{1}^{2}+\ldots \\
& u_{2}=\left(3-2 X_{2}\right)-\frac{\varepsilon}{3}\left(3-2 X_{2}\right)\left(c^{2}\left(X_{2}-1\right)\left(X_{2}-2\right)+3\right)+\ldots \\
& u_{3}=-1+\varepsilon\left(X_{3}-3\right)^{2}+\ldots
\end{aligned}
$$

where $c=\frac{c_{1}}{c_{2}}=\sqrt{\frac{E_{1} \rho_{2}}{E_{2} \rho_{1}}} \sim 1$. This coefficient appearing only at higher order incorporates the effect of the inertia of the soft component and the elastic properties of the stiff components. In fact, the dimensional leading order frequency $\omega^{2} \sim 2 E_{2} / l_{1}^{2} \rho_{1}$ in 41 depends only upon the density of the stiff component and 
the elastic properties of the soft component as in the approximate spring-mass model presented in the Appendix B.

The eigenforms (42) are shown in Figure 3 at $\varepsilon=0$ (solid line) and $\varepsilon=0.1$, so $c=1$ (dashed line). In this figure $u=u_{1}$ for $0 \leqslant x / l \leqslant 1, u=u_{2}$ for $1 \leqslant x / l \leqslant 2$, and $u=u_{3}$ for $2 \leqslant x / l \leqslant 3$.

It is worth noting that the leading order solution $\varepsilon=0$ associated with rigid body motion of stiff components and homogeneous deformation of soft components, justifies an elementary spring-mass model exposed in Appendix B.

${ }_{85}$ The correction to the leading order solution derived in this section reveals the deviation in the low-frequency behaviour of a continuous rod from the aforementioned discrete scheme. This observation is certainly true not only for a three-component rod.

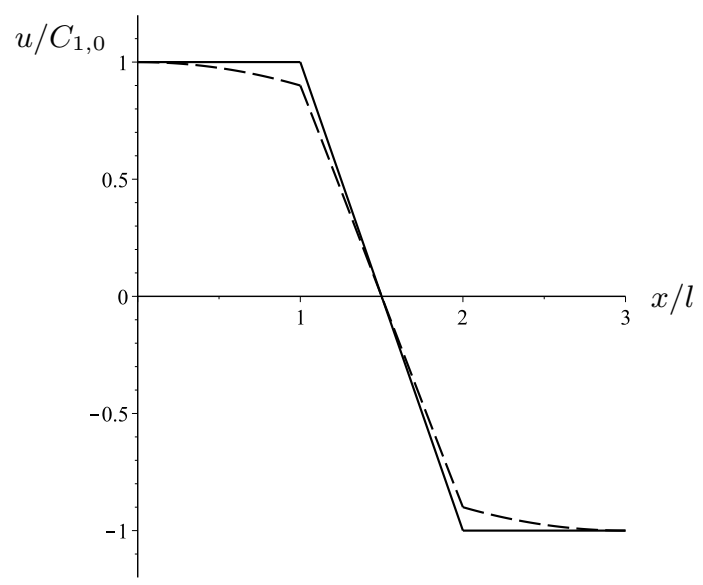

Figure 3: Asymptotic expansions 42 of the eigenform of a regular three-component rod at $\varepsilon=0$ (solid line) and $\varepsilon=0.1, c=1$ (dashed line).

\subsection{Five-component rod}

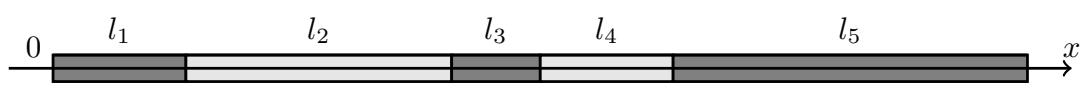

Figure 4: A five-component piecewise-homogeneous rod. 
Next, let us consider a five-component rod with three stiff and two soft components $\left(n_{1}=3, n_{2}=2\right)$, see Figure 4 . Then, we get from (31)

$$
\begin{aligned}
& C_{1,0} \Omega_{10}^{2}=L_{2}^{1}\left(C_{1,0}-C_{3,0}\right), \\
& C_{3,0} \Omega_{30}^{2}=\left(L_{2}^{3}+L_{4}^{3}\right) C_{3,0}-L_{2}^{3} C_{1,0}-L_{4}^{3} C_{5,0}, \\
& C_{5,0} \Omega_{50}^{2}=L_{4}^{5}\left(C_{5,0}-C_{3,0}\right)
\end{aligned}
$$

with

$$
\Omega_{10}=L_{3}^{1} \Omega_{30}=L_{5}^{1} \Omega_{50} .
$$

The formulae (43) together with relations (44), under the assumption $C_{3,0} \neq 0$ implies the following bi-cubic frequency equation

$$
\begin{aligned}
\Omega_{10}^{2}\left\{\Omega_{10}^{4}-\Omega_{10}^{2}\left(L_{2}^{1}+\right.\right. & \left.L_{2}^{1} L_{3}^{1}+L_{3}^{1} L_{4}^{1}+L_{4}^{1} L_{5}^{1}\right) \\
& \left.+L_{2}^{1} L_{4}^{1}\left(L_{3}^{1}+L_{3}^{1} L_{5}^{1}+L_{5}^{1}\right)\right\}=0
\end{aligned}
$$

resulting in 36$)$ where $k=0$, or

$$
\begin{aligned}
k=\frac{L_{5}^{1} L_{4}^{2}+L_{3}^{1}+L_{3}^{1} L_{4}^{2}+1}{2} & \\
& \pm \frac{\sqrt{\left(L_{5}^{1} L_{4}^{2}+L_{3}^{1}+L_{3}^{1} L_{4}^{2}+1\right)^{2}-4 L_{4}^{2}\left(L_{5}^{1}+L_{3}^{1}+L_{3}^{1} L_{5}^{1}\right)}}{2} .
\end{aligned}
$$

In addition, we deduce

$$
C_{3,0}=(1-k) C_{1,0}, \quad C_{5,0}=\frac{1-k}{1-L_{2}^{4} L_{1}^{5} k} C_{1,0}, \quad k \neq 1 .
$$

The associated eigenforms are

$$
\begin{aligned}
& u_{10}=C_{1,0}, \\
& u_{20}=C_{1,0}\left(1-k\left(X_{2}-b_{2}\right)\right), \\
& u_{30}=C_{1,0}(1-k), \\
& u_{40}=C_{1,0}(1-k)\left(1+\frac{L_{2}^{4} L_{1}^{5} k}{1-L_{2}^{4} L_{1}^{5} k}\left(X_{4}-b_{4}\right)\right), \\
& u_{50}=C_{1,0}(1-k) \frac{1}{1-L_{2}^{4} L_{1}^{5} k} .
\end{aligned}
$$

90 As for a three-component rod, these formulae are exact at $k=0$ corresponding to global rigid body motion. 
At next order, for $k$ given by $(46)$, we obtain corrections to the eigenfrequencies

$$
\begin{aligned}
\Omega_{11}^{2}=-\frac{1}{6}\left\{3 k(1-k) b_{3}^{2}\left(L_{2}^{3}\right)^{2}+k(3-k)(\right. & \left.c^{2}+\left(L_{2}^{1}\right)^{2}\right) \\
& \left.+6 L_{2}^{1}\left(C_{3,1}-k b_{3} L_{2}^{3}\right)\right\},
\end{aligned}
$$

together with the formulae

$$
\begin{aligned}
& u_{11}=A_{1,1} X_{1}^{2}, \\
& u_{21}=N_{2,1} X_{2}^{3}+F_{2,1} X_{2}^{2}+\left(G_{2,1}+C_{3,1}\right) X_{2}+H_{2,1}, \\
& u_{31}=A_{3,1} X_{3}^{2}+B_{3,1} X_{3}+C_{3,1}, \\
& u_{41}=N_{4,1} X_{4}^{3}+F_{4,1} X_{4}^{2}+\left(G_{4,1}+C_{5,1}-C_{3,1}\right) X_{4}+H_{4,1}, \\
& u_{51}=A_{5,1} X_{5}^{2}+B_{5,1} X_{5}+C_{5,1},
\end{aligned}
$$

where all the constants, apart from $C_{3,1}$ and $C_{5,1}$ can be determined from (24), (25), and 29]. Rather cumbersome expressions for $C_{3,1}$ and $C_{5,1}$, following from (31), are given in Appendix A.

In the particular case $C_{3,0}=0$, studied in greater detail below in this section for a regular five-component rod, we have $\Omega_{10}^{2}=L_{2}^{1}$ and necessarily the identity $L_{1}^{5} L_{2}^{5}=L_{4}^{5}$ must be satisfied. In this case eigenforms at leading order become

$$
\begin{aligned}
& u_{10}=C_{1,0}, \\
& u_{20}=C_{1,0}\left(1-\left(X_{2}-b_{2}\right)\right), \\
& u_{30}=0, \\
& u_{40}=-C_{1,0} L_{2}^{4}\left(X_{4}-b_{4}\right), \\
& u_{50}=-C_{1,0} L_{2}^{4} .
\end{aligned}
$$

95 Setting $C_{3,0}=0$ and $k=1$ in $(49)$ and (50), and taking into account $L_{1}^{5} L_{2}^{5}=L_{4}^{5}$, we can obtain the next order frequency and displacements for this particular case.

The eigenmodes for a regular five-component elastic $\operatorname{rod}\left(l_{i}=l, i=1,2, \ldots, 5\right)$ for $k=1$ and $k=3$ are respectively given by

$$
\Omega_{1}^{2}=\varepsilon-\frac{\varepsilon^{2}}{3}\left(c^{2}+\frac{5}{2}\right)+\ldots
$$


with

$$
\begin{aligned}
u_{1}= & 1-\frac{\varepsilon}{2} X_{1}^{2}+\ldots, \\
u_{2}= & \left(2-X_{2}\right)+\varepsilon\left(\frac{1}{6} c^{2} X_{2}^{3}-c^{2} X_{2}^{2}+\frac{11}{6} c^{2} X_{2}+X_{2}-c^{2}-\frac{3}{2}\right)+\ldots, \\
u_{3}= & \varepsilon\left(\frac{5}{2}-X_{3}\right)+\ldots, \\
u_{4}= & 3-X_{4} \\
& +\varepsilon\left(\frac{1}{6} c^{2} X_{4}^{3}-\frac{3}{2} c^{2} X_{4}^{2}+\frac{13}{3} c^{2} X_{4}+X_{4}-4 c^{2}-\frac{7}{2}\right)+\ldots, \\
u_{5}= & -1+\frac{\varepsilon}{2}\left(X_{5}-5\right)^{2}+\ldots,
\end{aligned}
$$

and

$$
\Omega_{1}^{2}=3 \varepsilon-\frac{\varepsilon^{2}}{2}\left(2 c^{2}+3\right)+\ldots,
$$

with

$$
\begin{aligned}
u_{1}= & -\varepsilon \frac{3}{2} X_{1}^{2}+\ldots, \\
u_{2}= & -3 X_{2} \\
& +\varepsilon\left(\frac{3}{2} c^{2} X_{2}^{3}-6 c^{2} X_{2}^{2}+\frac{17}{2} c^{2} X_{2}-4 c^{2}+3 X_{2}-\frac{9}{2}\right)+\ldots, \\
u_{3}= & -2+\varepsilon\left(c^{2}+3 X_{3}^{2}-15 X_{3}+\frac{39}{2}\right)+\ldots, \\
u_{4}= & 3 X_{4}-11 \\
& -\frac{\varepsilon}{2}\left(3 c^{2} X_{4}^{3}-33 c^{2} X_{4}^{2}+122 c^{2} X_{4}-152 c^{2}+6 X_{4}-21\right)+\ldots, \\
u_{5}= & -\varepsilon \frac{3}{2}\left(X_{5}-5\right)^{2}+\ldots
\end{aligned}
$$

The eigenforms (53) and (55) are displayed in Figure 5 for $c=1$. 


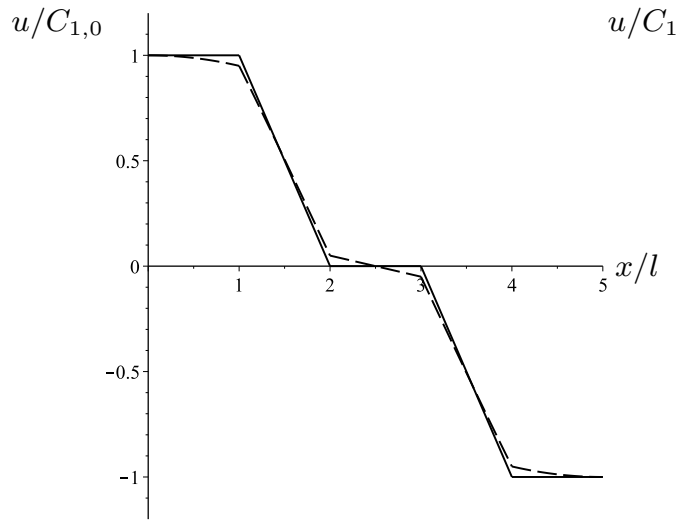

(a) $k=1$

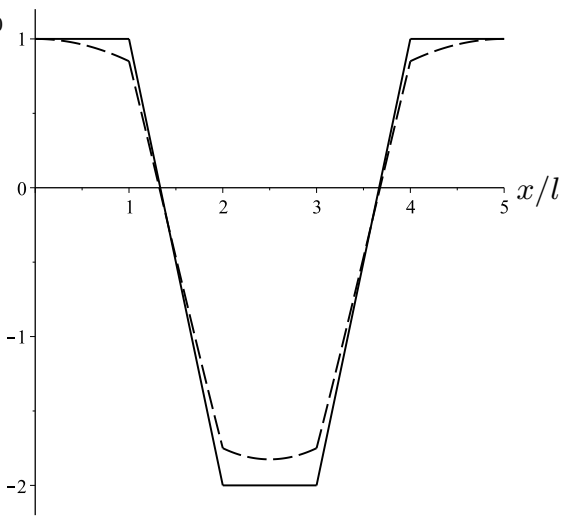

(b) $k=3$

Figure 5: Asymptotic expansions 53 (a) and 55 (b) of the eigenforms of a regular fivecomponent rod at $\varepsilon=0$ (solid line) and $\varepsilon=0.1, c=1$ (dashed line)

\section{Perturbation scheme for a multi-layered cylinder}

100 its layers. The cross-section shown in Figure 6, contains a hole of radius $l_{0}$, surrounded by a finite number $n$ of soft and stiff concentric annular layers of widths $l_{i}, i=1,2, \ldots, n$. Let us specify the small parameter $\varepsilon=\frac{\mu_{1}}{\mu_{2}} \ll 1$, where $\mu_{1}$ and $\mu_{2}$ are shear moduli in soft and stiff layers, respectively. Similarly to the

Consider a multi-layered hollow cylinder with high-contrast properties of previous consideration for a rod, we also assume that $\rho_{1} / \rho_{2} \sim \varepsilon$. 


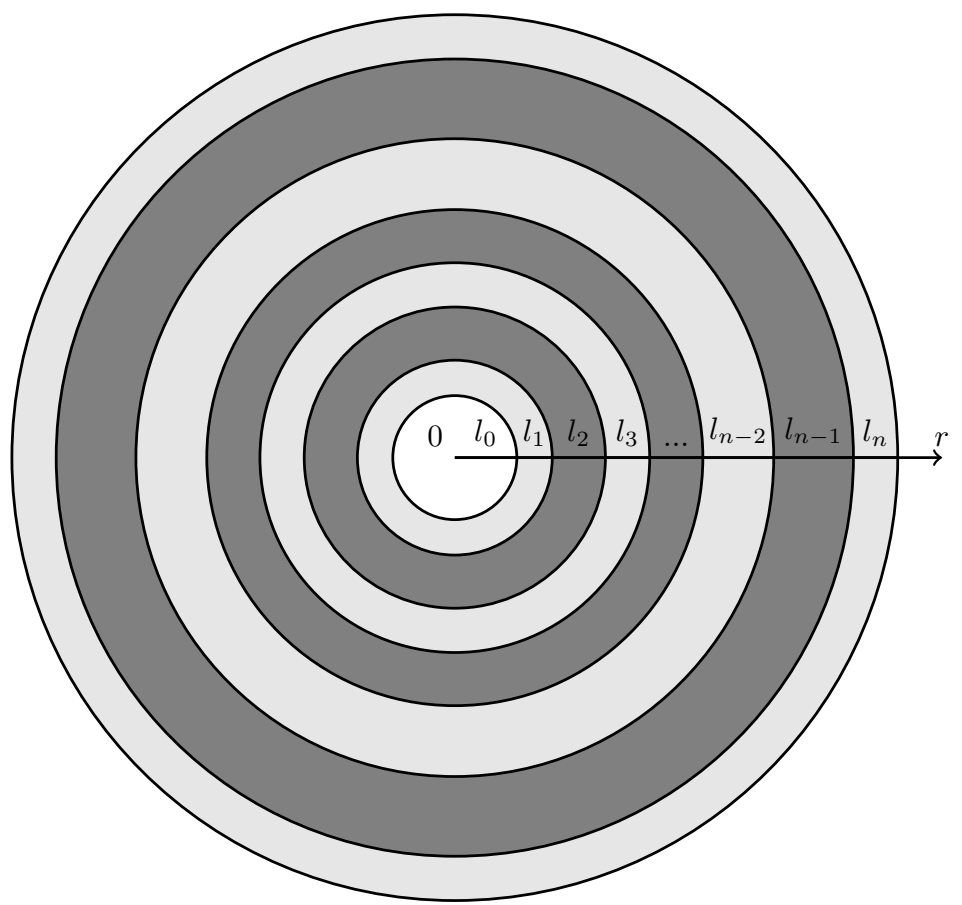

Figure 6: Cross-section of a hollow multi-layered circular cylinder.

The equations of anti-plane motion are written as

$$
r^{2} \frac{\mathrm{d}^{2} u_{i}}{\mathrm{~d} r^{2}}+r \frac{\mathrm{d} u_{i}}{\mathrm{~d} r}+\frac{\omega^{2} r^{2}}{c_{m}^{2}} u_{i}=0, \quad i=1,2, \ldots n, m=1,2,
$$

where $u_{i}$ denote out-of-plane displacements, $r$ is polar radius, and $c_{m}=\sqrt{\frac{\mu_{m}}{\rho_{m}}}$ are shear wave speeds.

First, we rewrite the equations (56) in dimensionless form as

$$
R_{i} \frac{\mathrm{d}^{2} u_{i}}{\mathrm{~d} R_{i}^{2}}+\frac{\mathrm{d} u_{i}}{\mathrm{~d} R_{i}}+\Omega_{i}^{2} R_{i} u_{i}=0
$$

with

$$
R_{i}=\frac{r}{l_{i}} \quad \text { and } \quad \Omega_{i}=\frac{\omega l_{i}}{c_{m}}
$$

The continuity conditions at interfaces are given by

$$
\left.u_{i}\right|_{R_{i}=b_{i}+1}=\left.u_{i+1}\right|_{R_{i+1}=b_{i+1}},
$$


and

$$
\left.\frac{\mathrm{d} u_{i}}{\mathrm{~d} R_{i}}\right|_{R_{i}=b_{i}+1}=\left.\varepsilon^{j} L_{i+1}^{i} \frac{\mathrm{d} u_{i+1}}{\mathrm{~d} R_{i+1}}\right|_{R_{i+1}=b_{i+1}},
$$

where $b_{i}$ are defined by (5), and $j=1$ if the $i$-th component is stiff, and $j=-1$ if it is a soft one.

In contrast to Section 2 for a rod, we restrict ourselves to fixed contours of the soft inner and outer layers of the cylinder, i.e.

$$
\left.u_{1}\right|_{R_{1}=b_{1}}=\left.u_{n}\right|_{R_{n}=b_{n}+1}=0 .
$$

Thus, we have now $n_{1}=\frac{n-1}{2}$ stiff layers and $n_{2}=\frac{n+1}{2}$ soft ones.

As before, we adapt the asymptotic series (10) and (11) over the global low-frequency range, i.e. $\Omega_{1}^{2} \sim \Omega_{2}^{2} \sim \ldots \sim \Omega_{n}^{2} \sim \varepsilon$.

At leading order, we get for the stiff layers the static equations

$$
R_{i} \frac{\mathrm{d}^{2} u_{i 0}}{\mathrm{~d} R_{i}^{2}}+\frac{\mathrm{d} u_{i 0}}{\mathrm{~d} R_{i}}=0, \quad i=2 p, \quad p=1,2, . ., n_{1},
$$

subject to the Neumann boundary conditions

$$
\left.\frac{\mathrm{d} u_{i 0}}{\mathrm{~d} R_{i}}\right|_{R_{i}=b_{i}}=\left.\frac{\mathrm{d} u_{i 0}}{\mathrm{~d} R_{i}}\right|_{R_{i}=b_{i}+1}=0 .
$$

The solutions of the formulated boundary value problems

$$
u_{i 0}=C_{i, 0}
$$

correspond to rigid body motions.

For the soft layers we now have the same equations 61 with $i=2 p-1, p=$ $1,2, \ldots, n_{2}$. These should be subject to a set of Dirichlet boundary conditions given by

$$
\begin{array}{ll}
\left.u_{i 0}\right|_{R_{i}=b_{i}}=C_{i-1,0}, & \left.u_{i 0}\right|_{R_{i}=b_{i}+1}=C_{i+1,0}, \\
\left.u_{10}\right|_{R_{1}=b_{1}}=0, & \left.u_{10}\right|_{R_{1}=b_{1}+1}=C_{2,0}, \\
\left.u_{n 0}\right|_{R_{n}=b_{n}}=C_{n-1,0}, & \left.u_{n 0}\right|_{R_{n}=b_{n}+1}=0 .
\end{array}
$$

The solution to the equations (61) with the boundary conditions $(64)$ gives for 
the soft layers

$$
\begin{aligned}
& u_{10}=C_{2,0} \delta_{1} \ln \left(\frac{R_{1}}{b_{1}}\right), \\
& \vdots \\
& u_{i 0}=C_{i-1,0}+\left(C_{i+1,0}-C_{i-1,0}\right) \delta_{i} \ln \left(\frac{R_{i}}{b_{i}}\right), \\
& \vdots \\
& u_{n 0}=C_{n-1,0}\left(1-\delta_{n} \ln \left(\frac{R_{n}}{b_{n}}\right)\right),
\end{aligned}
$$

where

$$
\delta_{i}=\frac{1}{\ln \left(1+b_{i}^{-1}\right)} .
$$

At next order, we write down for the stiff layers

$$
R_{i} \frac{\mathrm{d}^{2} u_{i 1}}{\mathrm{~d} R_{i}^{2}}+\frac{\mathrm{d} u_{i 1}}{\mathrm{~d} R_{i}}+\Omega_{i 0}^{2} R_{i} u_{i 0}=0, \quad i=2 p, \quad p=1,2, \ldots, n_{1},
$$

and

$$
\begin{aligned}
& \left.\frac{\mathrm{d} u_{i 1}}{\mathrm{~d} R_{i}}\right|_{R_{i}=b_{i}}=\frac{L_{i-1}^{i}\left(C_{i, 0}-C_{i-2,0}\right) \delta_{i-1}}{\left(b_{i-1}+1\right)}, \\
& \left.\frac{\mathrm{d} u_{i 1}}{\mathrm{~d} R_{i}}\right|_{R_{i}=b_{i}+1}=\frac{L_{i+1}^{i}\left(C_{i+2,0}-C_{i, 0}\right) \delta_{i+1}}{b_{i+1}} .
\end{aligned}
$$

Integrating (67), we deduce

$$
\left.R_{i} \frac{\mathrm{d} u_{i 1}}{\mathrm{~d} R_{i}}\right|_{R_{i}=b_{i}+1}-\left.R_{i} \frac{\mathrm{d} u_{i 1}}{\mathrm{~d} R_{i}}\right|_{R_{i}=b_{i}}=-C_{i, 0} \Omega_{i 0}^{2} \frac{2 b_{i}+1}{2} .
$$

Finally, using (68) and 69), we arrive at $(n>3)$

$$
\begin{aligned}
& C_{2,0} \Omega_{20}^{2}=\frac{2}{2 b_{2}+1}\left(C_{2,0} \delta_{1}+\left(C_{2,0}-C_{4,0}\right) \delta_{3}\right), \\
& \vdots \\
& C_{i, 0} \Omega_{i 0}^{2}=\frac{2}{2 b_{i}+1}\left(\left(C_{i, 0}-C_{i-2,0}\right) \delta_{i-1}+\left(C_{i, 0}-C_{i+2,0}\right) \delta_{i+1}\right), \\
& \quad \vdots \\
& C_{n-1,0} \Omega_{(n-1) 0}^{2}=\frac{2}{2 b_{n-1}+1}\left(\left(C_{n-1,0}-C_{n-3,0}\right) \delta_{n-2}+C_{n-1,0} \delta_{n}\right),
\end{aligned}
$$


where the frequency parameters are related to each other by the formulae (3).

115 Similarly to Section 2, the relations $(70)$ lead to a polynomial frequency equation of order $n_{1}=\frac{n-1}{2}$ in any of the frequency parameters $\Omega_{i 0}^{2}$. They also enable calculation of eigenforms together with the formulae 65 for the soft layers.

\section{Three- and five-layered cylinders}

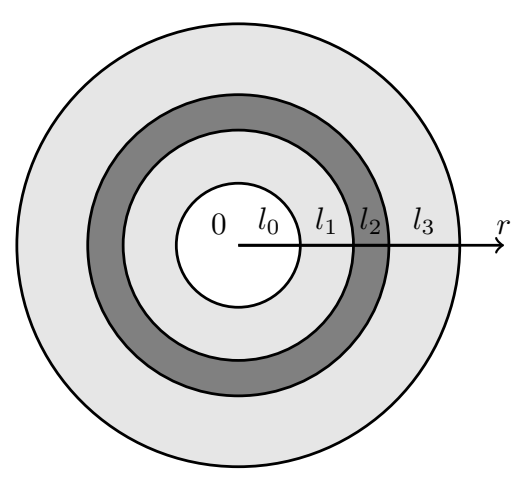

(a)

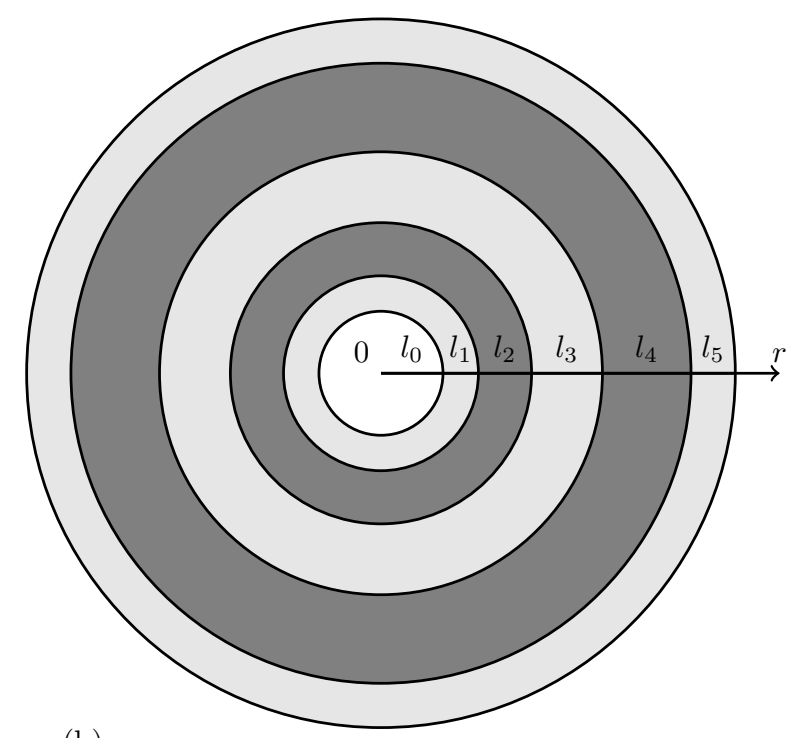

(b)

Figure 7: Cross-sections of a) three-layered and b) five-layered hollow circular cylinders.

Now, we can implement the formulae above to find the lowest eigenfrequencies and the associated eigenforms for anti-plane motion of a three-layered hollow circular cylinder, see Figure 7(a). Using (70), we can write down for the stiff layer

$$
\Omega_{20}^{2}=\frac{2\left(\delta_{1}+\delta_{3}\right)}{2 b_{2}+1}
$$


Hence, we have the following expressions for the leading order eigenforms

$$
\begin{aligned}
& u_{10}=C_{2,0} \delta_{1} \ln \left(\frac{R_{1}}{b_{1}}\right), \\
& u_{20}=C_{2,0}, \\
& u_{30}=C_{2,0} \delta_{3} \ln \left(\frac{b_{3}+1}{R_{3}}\right),
\end{aligned}
$$

demonstrated in Figure 8 for $l_{i}=l, i=0, \ldots, 3$ with $b_{1}=1, b_{3}=3, \delta_{1} \approx 1.44$, ${ }_{120} \quad \delta_{3} \approx 3.48$.

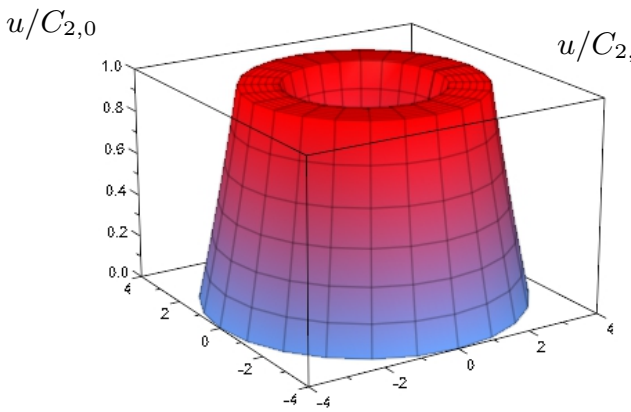

(a) 3D displacement profile

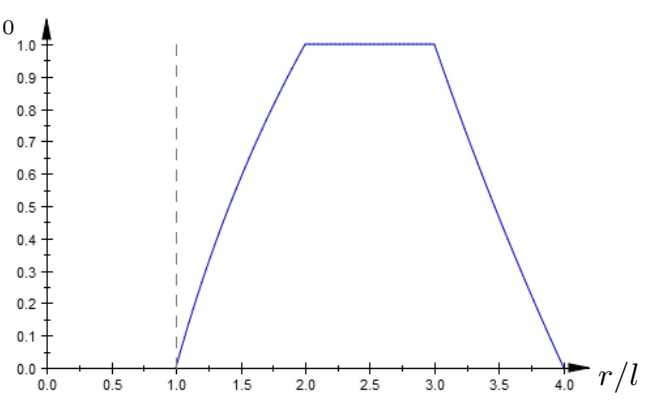

(b) $2 \mathrm{D}$ cross-section

Figure 8: Leading order approximation 72 of the eigenform of a three-layered cylinder with $l_{i}=l, i=0, \ldots, 3$ and $b_{1}=1, b_{3}=3, \delta_{1} \approx 1.44, \delta_{3} \approx 3.48$, (a) 3D profile and (b) $2 \mathrm{D}$ cross-section.

Next, for a five-component cylinder, see Figure 7(b), we get from 70 and $(3)$

$$
\begin{aligned}
& \Omega_{20}^{2}=\frac{2}{2 b_{2}+1}\left(\delta_{1}+\frac{\left(C_{2,0}-C_{4,0}\right) \delta_{3}}{C_{2,0}}\right), \\
& \Omega_{40}^{2}=\frac{2}{2 b_{4}+1}\left(\delta_{5}+\frac{\left(C_{4,0}-C_{2,0}\right) \delta_{3}}{C_{4,0}}\right),
\end{aligned}
$$

together with the relation

$$
\Omega_{20}=L_{4}^{2} \Omega_{40}
$$

Using the equations above, we can obtain the bi-quadratic frequency equa- 
tion

$$
\begin{aligned}
\Omega_{20}^{4}( & \left.L_{2}^{4}\right)^{2}\left(2 b_{2}+1\right)\left(2 b_{4}+1\right) \\
-2 \Omega_{20}^{2} & \left(\left(2 b_{2}+1\right)\left(\delta_{3}+\delta_{5}\right)+\left(L_{2}^{4}\right)^{2}\left(2 b_{4}+1\right)\left(\delta_{1}+\delta_{3}\right)\right) \\
& +4\left(\delta_{1} \delta_{3}+\delta_{3} \delta_{5}+\delta_{1} \delta_{5}\right)=0
\end{aligned}
$$

resulting in

$$
\Omega_{20}^{2}=\frac{2}{2 b_{2}+1}\left(\delta_{1}+(1-k) \delta_{3}\right),
$$

where the solutions for $k$ are

$$
\begin{aligned}
k=\frac{1}{2 \delta_{3}}\{ & \left(\left(\delta_{1}+\delta_{3}\right)-\gamma\left(\delta_{3}+\delta_{5}\right)\left(L_{4}^{2}\right)^{2}\right) \\
& \left. \pm \sqrt{\left(\left(\delta_{1}+\delta_{3}\right)-\gamma\left(\delta_{3}+\delta_{5}\right)\left(L_{4}^{2}\right)^{2}\right)^{2}+4 \gamma \delta_{3}^{2}\left(L_{4}^{2}\right)^{2}}\right\}
\end{aligned}
$$

with

$$
\gamma=\frac{2 b_{2}+1}{2 b_{4}+1} .
$$

As a result, the leading order eigenforms are

$$
\begin{aligned}
& u_{10}=C_{2,0} \delta_{1} \ln \left(\frac{R_{1}}{b_{1}}\right), \\
& u_{20}=C_{2,0}, \\
& u_{30}=C_{2,0} \delta_{3}\left\{k \ln \left(\frac{R_{3}}{b_{3}}\right)-\ln \left(\frac{R_{3}}{b_{3}+1}\right)\right\}, \\
& u_{40}=C_{2,0} k, \\
& u_{50}=C_{2,0} k \delta_{5} \ln \left(\frac{b_{5}+1}{R_{5}}\right) .
\end{aligned}
$$

It is clear that in the considered case of two stiffer layers, there are two options for uniformly varying leading order displacements $u_{20}$ and $u_{40}$, being either of the same or opposite sign. The associated numerical illustrations are presented in Figure 9 for $k \approx 0.74$ and in Figure 10 for $k \approx-0.75$ with $l_{i}=l, i=0, \ldots, 5$, ${ }_{125} b_{1}=1, b_{3}=3, b_{5}=5, \delta_{1} \approx 1.44, \delta_{3} \approx 3.48, \delta_{5} \approx 5.48$. 
$u / C_{2,0}$

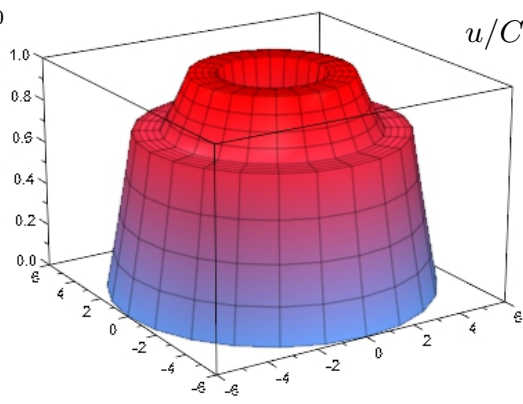

(a) 3D displacement profile

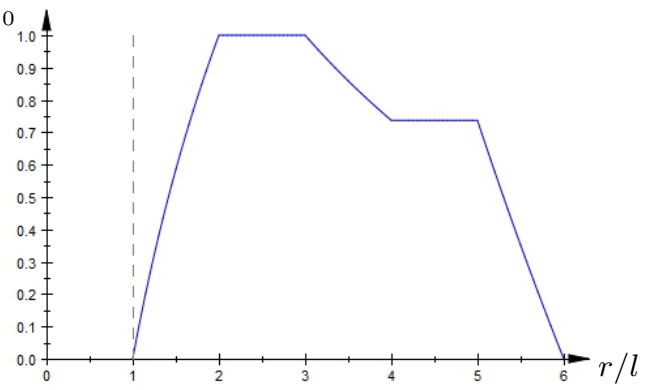

(b) $2 \mathrm{D}$ cross-section

Figure 9: Leading order approximation 777 of the eigenform of a five-layered cylinder with $k \approx 0.74$ and $l_{i}=l, i=0, \ldots, 5, b_{1}=1, b_{3}=3, b_{5}=5, \delta_{1} \approx 1.44, \delta_{3} \approx 3.48, \delta_{5} \approx 5.48$, (a) $3 \mathrm{D}$ profile and (b) $2 \mathrm{D}$ cross-section.

$u / C_{2,0}$

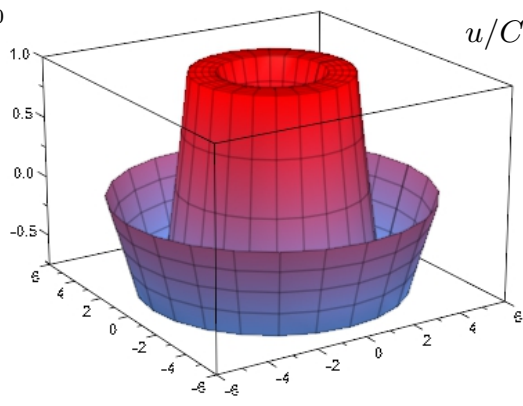

(a) 3D displacement profile

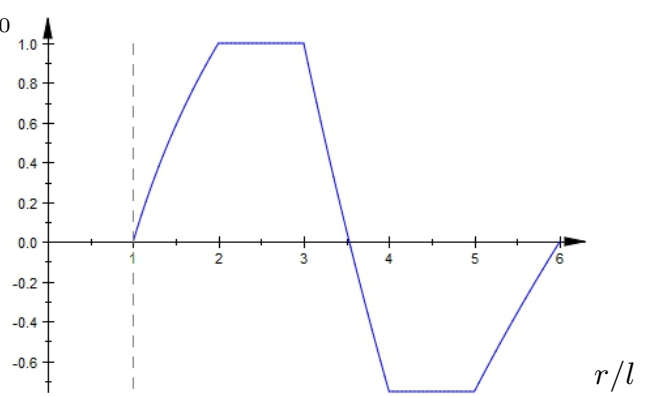

(b) $2 \mathrm{D}$ cross-section

Figure 10: Leading order approximation 777 of the eigenform of a five-layered cylinder with $k \approx-0.75$ and $l_{i}=l, i=0, \ldots, 5, b_{1}=1, b_{3}=3, b_{5}=5, \delta_{1} \approx 1.44, \delta_{3} \approx 3.48, \delta_{5} \approx 5.48$, (a) $3 \mathrm{D}$ profile and (b) $2 \mathrm{D}$ cross-section.

\section{Concluding remarks}

The leading order approximations for the eigenfrequencies and eigenforms of multi-component strongly inhomogeneous rods and cylinders are established, see (22) and (70), respectively, as well as the first order corrections (31) in case of rods. 
The example of a three-component rod in subsection 3.1 justifies at leading order the validity of the elementary mass-spring scheme, exposed in Appendix B. The deviation of the discrete formulation from the continuous model is observed at next order, when the elastic properties of the stiff components and inertia of the soft ones, neglected within the above mentioned discrete setup, are taken into account.

The developed approach may be extended to multi-parametric problems, e.g. strong variation in linear sizes of components, leading to local low-frequency regimes, as in [11, characterized by oscillatory behaviour of softer components. More elaborate systems involving beams and plates can also be tackled, for which, in addition to translational almost rigid body motion studied in this paper, rotational ones occur.

It should be emphasized that within the exact formulation of the problem the frequency equation is always given by a nontrivial transcendental relation involving oscillating trigonometric functions and Bessel functions for rods and cylinders, respectively.

The obtained results are of interest for low-frequency analysis in multilayered elastic plates and shells [14, [15, [16, and also for investigation of buckling phenomena in multi-span engineering structures, e.g. see [17, due to the well-known similarity of the eigenvalue problems in vibration and stability theories. The presented methodology may be implemented to a more general layout as well as other types of boundary conditions at the ends of the multicomponent structures. This may also be exploited in scalar 2D, 3D, and vector problems. Finally, we note potential applications to robotics, especially in view of the recent developments of soft robots [18 and hybrid systems [19. In this case, analysis of the lowest vibration modes caused by the presence of soft components bringing additional degrees of freedom seems to be relevant. 


\section{Acknowledgement}

This work was supported by the grant J2-9224 from the Slovenian Research acknowledges support by the Faculty Award by Keele University, UK.

\section{Appendix A}

The constant $C_{3,1}$ for a three-component rod is given by

$$
C_{3,1}=\frac{2}{L_{2}^{3}-\Omega_{30}^{2}+L_{1}^{3} L_{2}^{3}(1-k)}\left(3\left(\Omega_{30}^{2} P_{3}-L_{2}^{3} Q_{2}\right)-(1-k) L_{1}^{3}\left(3 L_{2}^{3} P_{2}+A_{1,1} \Omega_{10}^{2} L_{1}^{3}\right)\right) .
$$

For a five-component rod the constants $C_{3,1}$ and $C_{5,1}$ take the form

$$
\begin{aligned}
C_{3,1} & =\frac{\left(1-L_{2}^{4} L_{1}^{5} k\right) C_{1,0}}{P_{1}}\left(6 \Omega_{50}^{2}\left(L_{2}^{3} Q_{2}-\Omega_{30}^{2} P_{3}+L_{4}^{3}\left(P_{5}-P_{4}\right)\right)\right. \\
& -6 L_{4}^{5}\left(L_{2}^{3} Q_{2}+L_{4}^{3} S_{4}-\Omega_{30}^{2} P_{3}\right)+2(1-k)\left\{L_{1}^{3}\left(\Omega_{50}^{2}-L_{4}^{5}\right)\left(3 L_{2}^{3} P_{2}+A_{1,1} L_{1}^{3} \Omega_{10}^{2}\right)\right. \\
& \left.\left.-\frac{L_{1}^{5} L_{4}^{3}}{1-L_{2}^{4} L_{1}^{5} k}\left(3 L_{2}^{5} P_{2}+A_{1,1} L_{1}^{5} \Omega_{10}^{2}\right)\right\}\right)
\end{aligned}
$$

and

$$
\begin{aligned}
C_{5,1} & =\frac{\left(1-L_{2}^{4} L_{1}^{5} k\right) C_{1,0}}{P_{1}}\left(L_{4}^{5}\left(6 \Omega_{30}^{2}\left(Q_{4}+P_{3}\right)-6 L_{2}^{3}\left(Q_{4}+P_{2}+S_{2}\right)-6 L_{4}^{3} S_{4}\right)\right. \\
& +6 P_{5} \Omega_{50}^{2}\left(L_{2}^{3}+L_{4}^{3}-\Omega_{30}^{2}\right)-2(1-k) L_{1}^{3}\left(L_{4}^{5}\left(3 L_{2}^{3}\left(Q_{4}+P_{2}\right)+A_{1,1} L_{1}^{3} \Omega_{10}^{2}\right)-3 L_{2}^{3} \Omega_{50}^{2} P_{5}\right) \\
& -\frac{2(1-k) L_{1}^{5}}{1-L_{2}^{4} L_{1}^{5} k}\left(L_{2}^{3}\left(A_{1,1} L_{1}^{5} \Omega_{10}^{2}-3 L_{2}^{5} S_{2}\right)\right. \\
& \left.\left.-\Omega_{30}^{2}\left(A_{1,1} L_{1}^{5} \Omega_{10}^{2}+3 L_{2}^{5}\left(P_{2}-P_{3}\right)\right)+L_{4}^{3}\left(A_{1,1} L_{1}^{5} \Omega_{10}^{2}+3 L_{2}^{5}\left(P_{2}+P_{4}\right)\right)\right)\right) .
\end{aligned}
$$


In the above

$$
\begin{aligned}
& P_{1}= 6 C_{1,0}\left\{\left(1-L_{2}^{4} L_{1}^{5} k\right)\left(L_{4}^{5}\left(L_{2}^{3}-\Omega_{30}^{2}\right)-\Omega_{50}^{2}\left(L_{2}^{3}+L_{4}^{3}-\Omega_{30}^{2}\right)\right)\right. \\
&\left.\quad+(1-k)\left(1-L_{2}^{4} L_{1}^{5} k\right)\left(L_{1}^{3} L_{2}^{3} L_{4}^{5}-L_{1}^{3} L_{2}^{3} \Omega_{50}^{2}\right)+(1-k) L_{1}^{5} L_{2}^{5} L_{4}^{3}\right\} \\
& P_{i}=3 N_{i, 1} b_{i}^{2}+2 F_{i, 1} b_{i}+G_{i, 1}, \quad i=2,4, \\
& P_{j}=A_{j, 1}\left(b_{j}^{2}+b_{j}+\frac{1}{3}\right)+B_{j, 1}\left(b_{j}+\frac{1}{2}\right), \quad j=3,5, \\
& Q_{i}=3 N_{i, 1}\left(b_{i}+1\right)^{2}+2 F_{i, 1}\left(b_{i}+1\right)+G_{i, 1}, \quad i=2,4, \\
& S_{i}=3 N_{i, 1}\left(2 b_{i}+1\right)+2 F_{i, 1}, \quad i=2,4,
\end{aligned}
$$

and all the constants $A_{i, 1}, B_{i, 1}, N_{i, 1}, F_{i, 1}, G_{i, 1}$, and $H_{i, 1}$ are defined from 24, (25), and (29).

\section{Appendix B}

Consider a mass-spring system, see Figure 11, with two masses $m_{1}$ and $m_{2}$, connected by an elastic spring of stiffness $c$, as an approximate discrete model for a three-component rod treated in subsection 3.1, see Figure 2.

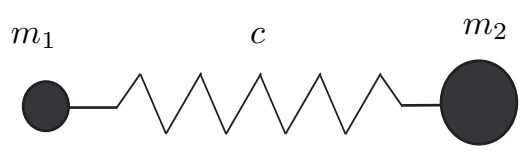

Figure 11: Mass-sping system

In this case we suppose

$$
m_{1}=\rho_{1} l_{1} A, \quad m_{2}=\rho_{1} l_{3} A, \quad c=\frac{E_{2} A}{l_{2}},
$$

where $A$ is the rod's cross-section, see e.g. [20].

Let the coordinates of the centres of masses be $z_{1}$ and $z_{2}$. Then, the equations of time-harmonic motion are

$$
\begin{aligned}
& \left(c-m_{1} \omega^{2}\right) z_{1}-c z_{2}=0, \\
& c z_{1}-\left(c-m_{2} \omega^{2}\right) z_{2}=0,
\end{aligned}
$$


from which the eigenfrequency $\omega$ is either zero, or

$$
\omega^{2}=\frac{m_{1}+m_{2}}{m_{1} m_{2}} c,
$$

and the related eigenform is

$$
z_{1}=\alpha, \quad z_{2}=-\frac{m_{1}}{m_{2}} \alpha,
$$

where $\alpha$ is an arbitrary constant.

In terms of the parameters of the rod $(\bar{B} .1)$ the formulae $(\mathrm{B} .3)$ and $(\mathrm{B} .4)$ become

$$
\omega^{2}=\frac{\left(l_{1}+l_{3}\right) E_{2}}{\rho_{1} l_{1} l_{2} l_{3}},
$$

and

$$
z_{1}=\alpha, \quad z_{2}=-\frac{l_{1}}{l_{3}} \alpha,
$$

respectively.

It may be easily verified that the leading order approximation $\Omega_{10}^{2}$, see $(36)$, expressed in dimensional form by using (2) and (10), is identical to (B.5). Also, the leading order approximation for the eigenform for a three-component rod

175 (38) may be interpreted using the formula (38) by setting in the former $C_{1,0}=\alpha$, and $k=1+L_{3}^{1}$.

Finally, we mention that the presented mass-spring model does not incorporate the effect of the elastic properties of the stiff components of the rod, and inertia of the soft ones. The latter appear at the next order approximation, deduced in section 3.1 .

\section{References}

[1] M. Aßmus, K. Naumenko, H. Altenbach, Mechanical behaviour of photovoltaic composite structures: Influence of geometric dimensions and material properties on the eigenfrequencies of mechanical vibrations, Composites 
[2] K. Viverge, C. Boutin, F. Sallet, Model of highly contrasted plates versus experiments on laminated glass, International Journal of Solids and Structures 102 (2016) 238-258. doi:10.1016/j.ijsolstr.2016.09.035.

[3] Y. Qin, X. Wang, Z. L. Wang, Microfibre-nanowire hybrid structure for energy scavenging, Nature 451 (7180) (2008) 809-813. doi:10.1038/ nature06601.

[4] M. Ruzzene, A. Baz, Attenuation and localization of wave propagation in periodic rods using shape memory inserts, Smart Materials and Structures 9 (6) (2000) 805-816.

[5] T. P. Martin, C. N. Layman, K. M. Moore, G. J. Orris, Elastic shells with high-contrast material properties as acoustic metamaterial components, Physical Review B 85 (16) (2012) 161103. doi:10.1103/PhysRevB. 85.161103 .

[6] A. S. Sayyad, Y. M. Ghugal, Bending, buckling and free vibration of laminated composite and sandwich beams: A critical review of literature, Composite Structures 171 (2017) 486-504. doi:10.1016/j.compstruct.2017. 03.053

[7] M. Cherdantsev, K. D. Cherednichenko, Two-scale $\Gamma$-convergence of integral functionals and its application to homogenisation of nonlinear highcontrast periodic composites, Archive for Rational Mechanics and Analysis 204 (2012) 445-478. doi:10.1007/s00205-011-0481-4

[8] V. P. Smyshlyaev, Propagation and localization of elastic waves in highly anisotropic periodic composites via two-scale homogenization, Mechanics of Materials 41 (4) (2009) 434-447. doi:10.1016/j.mechmat.2009.01.009.

[9] J. Kaplunov, A. Nobili, Multi-parametric analysis of strongly inhomogeneous periodic waveguides with internal cut-off frequencies, Mathemat-

口 ical Methods in the Applied Sciences 40 (9) (2017) 3381-3392. doi: $10.1002 / \mathrm{mma} .3900$. 
[10] M. Cherdantsev, K. Cherednichenko, S. Cooper, Extreme localisation of ijsolstr.2017.01.042

[15] L. Prikazchikova, Y. Ece Aydın, B. Erbaş, J. Kaplunov, Asymptotic anal-

[16] P. E. Tovstik, T. P. Tovstik, Generalized Timoshenko-Reissner models for beams and plates, strongly heterogeneous in the thickness direction,

Angewandte Mathematik und Mechanik 97 (3) (2017) 296-308. doi: 10.1002/zamm. 201600052 . 
[17] Y. Li, I. Elishakoff, J. Starnes Jr, Buckling mode localization in a multispan periodic structure with a disorder in a single span, Chaos, Solitons \& Fractals 5 (6) (1995) 955-969. doi:10.1016/0960-0779(94)00211-8.

[18] D. Rus, M. T. Tolley, Design, fabrication and control of soft robots, Nature 521 (7553) (2015) 467-475. doi:10.1038/nature14543.

[19] A. A. Stokes, R. F. Shepherd, S. A. Morin, F. Ilievski, G. M. Whitesides, A hybrid combining hard and soft robots, Soft Robotics 1 (1) (2014) 70-74. doi:10.1089/soro.2013.0002.

[20] T.-R. Hsu, Applied Engineering Analysis, Hoboken, NJ: John Wiley \& Sons, 2018. 